\title{
Commemoration of Steve R. Goldberg; 1941-2014; age 73
}

\author{
Jack Bergman ${ }^{1}$
}

Received: 4 April 2016/Accepted: 4 April 2016/Published online: 11 April 2016

(C) Springer-Verlag Berlin Heidelberg 2016

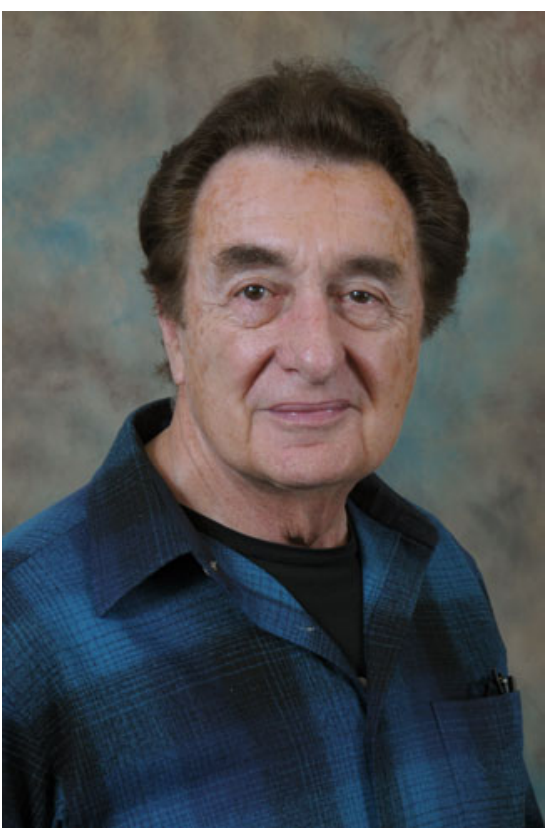

Steven R. Goldberg died unexpectedly of cardiac failure on 25 November 2014. Steve is survived by his siblings James and Elaine, his wife Sevil Yasar, and their children Sebastian and Margit. Death came while Steve was exercisingcertainly, a grim irony that he would have appreciated

Jack Bergman

jbergman@hms.harvard.edu

1 Behavioral Biology Laboratory, McLean Hospital / Harvard Medical School, Belmont, MA, USA intellectually. With his passing, Steve's family and his many friends and colleagues lost someone whose usually noneffusive exterior belied a warmth and generosity that knew no boundaries. He cared deeply for those around him, especially family and young colleagues who depended upon him for emotional and professional support and to whom he felt the intrinsic obligation to provide that support. In the course of his rich life, Steve also fashioned an illustrious, 5-decade-long scientific career that has had an outsized influence on our understanding of the behavioral and pharmacological determinants of reinforcing and other effects of psychoactive drugs.

Steve grew up in Boston, MA, and received his undergraduate degree in Biology at Northeastern University in 1964, after which he moved to the University of Michigan for graduate training in pharmacology. There, working with Bob Schuster on morphine self-administration and opiate withdrawal, he became intrigued by the interplay of Pavlovian and operant conditioning factors in the reinforcing effects of drugs and drug dependence, a fascination that he maintained throughout the entirety of his career. In that early work, Steve showed that the injection of opioid antagonists or stimuli conditioned to the injection could increase morphine selfadministration in morphine-dependent monkeys and produce other behavioral and physiological signs of opioid withdrawal. Moreover, injections of nalorphine, which provoked signs of withdrawal in morphine-dependent monkeys, continued to do so even in post-dependent monkeys. In conjunction, Steve's findings provided powerful evidence regarding the role of withdrawal and withdrawal-related conditioning factors in the reinforcing effects of morphine after, as well as during, opioid dependence. Steve furthered his interest in conditioning during a postdoctoral year with Neil Schneiderman at the University of Miami and then, in 1970, moved to the Psychobiology Laboratory at Harvard Medical School. For most of the next decade, Steve, in concert with Bill Morse, 
Roger Kelleher, and Roger Spealman, conducted extensive studies in nonhuman primates, forcefully demonstrating the power of schedules and conditioning in controlling the reinforcing and other effects of different classes of selfadministered drugs. His work with Roger Spealman using second-order schedules to develop robust nicotine selfadministration was instrumental to the declaration in 1988 by the U.S. Surgeon General, Dr. C. Everett Koop, that nicotine, like cocaine or heroin, was an addictive drug - which, in turn, was the first step in combating tobacco use in the USA. Overall, the body of work that resulted from the research conducted by Steve and his colleagues during his years at Harvard continues to guide our understanding of the behavioral pharmacology of opioids and psychomotor stimulants, especially cocaine and nicotine.

In 1979, Steve left the Psychobiology Laboratory to move to the Addiction Research Center in Lexington, Kentucky, which was soon transformed into the Intramural Research Program (IRP) of the National Institute on Drug Abuse in Baltimore, Maryland. Years later, Steve allowed that, though he would have been content to stay at Harvard, the move had worked out remarkably well for the development of his career. And what a career it was! For the next 35 years in Baltimore, Steve thrived: he continued to work with psychomotor stimulants and, in a highly influential paper in Science in 1987, he, along with Mike Kuhar and Mary Ritz, forwarded the now well-accepted view that the reinforcing effects of cocaine were mediated through blockade of the dopamine transporter. He also continued to work with nicotine and showed that, as in his previous work in monkeys, i.v. nicotine maintained exemplary schedule-controlled responding in human subjects. And, in what has proven to be a remarkably productive endeavor over the past 15 years, he initiated a program of research into cannabinoid pharmacology. As had been the case with nicotine, it was difficult at the time to demonstrate that $\Delta^{9}$-THC, the major active constituent of marijuana, had reinforcing effects in laboratory animals.
As with nicotine, Steve willingly embraced this challenge. After considerable effort, he and his colleagues were able to identify the factors that were key to the self-administration of $\Delta^{9}$-THC and showed that it, as well as endocannabinoids, could have robust reinforcing effects in nonhuman primates. This was an achievement that depended upon Steve's unrivaled understanding of the interplay of environmental, historical, and pharmacological factors in the reinforcing effects of drugs.

Throughout his years at the IRP, Steve built a laboratory that was continuously productive and vibrant. Steve Goldberg was an internationalist. He collaborated extensively with foreign scientists and, as a result, there was a constant flow of postdoctoral fellows from abroad to his laboratory. He loved to travel and he appreciated exposure to diverse cultures; bringing in young scientists from abroad imparted a youthful and international flavor that he thoroughly enjoyed in his daily life. As Steve's research focus broadened over the years, he also expanded his scientific approach. He began to undertake collaborations with scientists within and outside the IRP in multidisciplinary efforts to developing candidate therapeutics or to understanding neurobiological mechanisms in the behavioral effects of drugs. Perhaps to his own surprise, Steve was fantastic in this role. He enjoyed bringing scientists together to work on a problem and then, in concert, weaving the strands of experimental evidence into a narrative that explained the original observations and that could foster further research. Most of all, he enjoyed the interpersonal relationships these professional interactions provided - and if they included foreign collaboration, so much the better. He thrived on constant contact with people from various cultures and countries, and this led to an empathy that was reflected in his sensibilities and his compassion. Steve would have immensely appreciated the effort that led, first, to last year's commemorative symposium at the IRP and now, this volume of work honoring his professional contributions and reflecting his broad impact. He will continue to be missed by everybody who knew him well. 